\title{
Effects of Microgravity on the Monocyte/Macrophage Physiology and the Potential Implication in Immune Responses to Vaccines
}

\author{
Jiawei Yang (D), Wanlin Xing (D), Wang Chongzhen* \\ Guangxi Provincial Key Laboratory of Diabetic Systems Medicine, Guilin Medical University, Guilin, China
}

\begin{abstract}
A R T ICLE IN F O
A B S T R A C T

Review Article

VacRes, 2019

Vol. 7, No.1, 47-52

Received: March 03, 2020

Accepted:June 22, 2020

Pasteur Institute of Iran

*Corresponding Author: Dr. Chongzhen Wang, Guangxi Provincial Key Laboratory of Diabetic Systems Medicine, Guilin Medical University, 109 Huan Cheng Bei Er Lu, Guilin 541004, China.

Email: 022101043@ fudan.edu.cn

Tel/Fax: +86 773-368-0353/+86 773$589-5881$

KEYWORDS: Microgravity,

Monocyte, Macrophage

Introduction: Exposure to the microgravity environment could cause human physiological abnormalities that include fluid shift, anemia, osteoporosis, immunosuppression, etc. Monocytes and macrophages are important components of the immune system and could serve as precursors of osteoclasts. A direct effect of microgravity on monocytes and/or macrophages may contribute to immunosuppression and osteoporosis. Methods: To test this hypothesis, we reviewed the studies concerning the direct effects of microgravity on the monocyte/macrophage physiology and discussed the links of these effects to the human physiological abnormalities caused by microgravity. Results: Inhibited development and proliferation, reduced production of reactive oxygen species and proinflammatory cytokines and inhibited cell locomotion were observed in monocyte/macrophages under microgravity. These microgravity effects may lead to immunosuppression. Enhanced osteoclastogenesis was observed in monocyte/macrophages under microgravity. This microgravity effect may lead to osteoporosis. The molecular mechanisms underlying these microgravity effects were also presented. For example, the mechanisms of the inhibited cell locomotion under microgravity include: reduced $\beta$-actin expression, abnormality in the structure of focal adhesions and impaired protein kinase $\mathrm{C}$ signaling. Conclusion: The elucidation of the molecular mechanisms by which microgravity interferes with the monocyte/macrophage physiology may favor the identification of potential drug targets to reverse the deleterious effects of microgravity. Furthermore, due to the fact that macrophages are professional antigen-presenting cells, we propose that microgravity might modulate the efficacy of antigen presentation by macrophages in the immune response to vaccines.

\section{Citaion:}

Yang J, Wang C, Xing W. Effects of Microgravity on the Monocyte/Macrophage Physiology and the Potential Implication in Immune Responses to Vaccines. vacres. 2019; 6 (2) :47-52. DOI: 10.29252/vacres.6.2.47
\end{abstract}

\section{INTRODUCTION}

To travel into the space, humans need to adapt to microgravity in space. Unfortunately, exposure to the microgravity environment could cause severe abnormalities in the human physiology, which include fluid shift, anemia, osteoporosis, immunosuppression, etc [1, 2]. Monocytes and macrophages are important components of the immune system and could serve as precursors of osteoclasts that are responsible for the bone resorption. Modulation of the monocyte/macrophage physiology by microgravity might underlie the immunosuppression and osteoporosis of humans in space. In the present review, we summarize the effects of microgravity on the development, general physiology, immunerelated functions and osteoclast-directed differentiation of monocyte/macrophages, and discuss the links of these effects to the human physiological abnormalities caused by microgravity.
Considering that macrophages are professional antigenpresenting cells, microgravity might also affect their efficacy of antigen presentation during the immune response to vaccines.

\section{MATERIALS and METHODS}

In the search box of PubMed, the following terms were typed: ("Weightlessness Simulation"[Mesh] OR "Weightlessness"[Mesh]) AND ("Macrophages"[Mesh] OR "Monocytes"[Mesh]). PubMed Search was run. Studies concerning the direct effects of microgravity on the monocyte/macrophage physiology were selected. These microgravity effects were classified in 5 sections: "Development and general physiology", "Cytokine secretion", "Production of reactive oxygen species (ROS)", "Cell migration", and "Osteoclastogenesis". The molecular 
mechanisms underlying these microgravity effects were presented as well.

\section{RESULTS}

\section{Development and General Physiology}

Monocytes and macrophages are derived from hematopoietic stem cells. Macrophage colony-stimulating factor (M-CSF) could induce the differentiation of bone marrow cells into macrophages. This differentiation process are reported to be inhibited in space [3]. Furthermore, the total Kupffer cell (liver macrophage) population in spaceflight rats appear to be reduced [4]. These data suggest that microgravity might impair macrophage development. In the macrophages differentiated from bone marrow cells under microgravity, the expressions of genes in the RAS/ERK/NF-KappaB signaling pathway are shown to be down-regulated [5]. Furthermore, both an ERK agonist and an NF-KappaB agonist can rescue the impaired macrophage development under microgravity [5]. Thus, microgravity might impair macrophage development through down-regulating the expression of genes in the ERK and NF-KappaB signaling pathways.

Cytoskeleton structures of microfilaments, microtubules and focal adhesion plaques are reported to be altered in J111 cells (a human monocyte/macrophage cell line) cultured under no matter real or simulated microgravity $[6,7]$. In addition, the intensity of F-actin and $\beta$-tubulin immunofluorescence is shown to be reduced in the microgravity-exposed J111 cells [6]. Consistently, the amount of $\beta$-actin, which is a major component of the F-actin microfilaments, is reduced in U937 cells (a human monocytic cell line) cultured under simulated microgravity [8]; and the amount of $\alpha$-tubulin, which heterodimerizes with $\beta$-tubulin to form the basic unit of microtubules, is reduced in FLG 29.1 cells (a human monocytic cell line that could be induced to differentiate into osteoclastlike cells [9]) cultured under simulated microgravity [10]. Interestingly, the expression of vimentin, an intermediate filament protein, is shown to be increased in the microgravityexposed FLG 29.1 cells [10]. The functional significance of the increased vimentin expression under microgravity requires further investigation.

When exposed to no matter real or simulated microgravity, U937 cells grow slower [11, 12, 8]. The protein amount of cdc25B, a cell cycle regulator essential for the $\mathrm{G} 2 / \mathrm{M}$ phase transition in human cells [13], is documented to be significantly decreased in U937 cells cultured under simulated microgravity [8]. This might lead to growth retard of U937 cells at the G2 phase under simulated microgravity [8]. Furthermore, microtubules and microfilaments play important roles in cell cycle progression, which are responsible for the alignment and segregation of chromosomes [14], and the contraction and abscission at the cell division plane (cytokinesis) [15], respectively. Abnormalities in the expression and structure of microfilaments and microtubules as mentioned above, may also contribute to the reduced growth under microgravity.

Unlike lymphocytes, U937 cells cultured under simulated microgravity do not undergo apoptosis $[16,8]$. Activation of 5LOX (5-lipoxygenase) is found to be the cause of lymphocyte apoptosis under the microgravity environment. Lack of 5-LOX expression may protect U937 cells from apoptosis under microgravity [16]. In addition, the major stress protein Hsp70 (the 70 kilodalton heat shock proteins) is shown to be upregulated in U937 cells, under microgravity which could also protect the cells from apoptosis [8]. However, culturing the preosteoclastic FLG 29.1 cells with bone slices under microgravity results in a marked increase in apoptosis compared with under $1^{*} \mathrm{~g}$ condition [10]. It is possible that the bone slices in the culture could influence the expression of 5LOX and/or Hsp70 in FLG 29.1 cells and sensitize the cells to microgravity-mediated apoptosis.

The cell-surface protein, Intercellular Adhesion Molecule1 (ICAM-1), is found to be a gravity-regulated molecule in macrophage cells. Its expression in murine BV-2 microglial cells is shown to be down-regulated by microgravity. Furthermore, the ICAM-1 expression in the macrophage-like differentiated human U937 cells is up-regulated by microgravity [17]. Recently, we found that the Major Histocompatibility Complex (MHC) class II molecule, I-A ${ }^{\mathrm{b}}$, in primary mouse macrophages is down-regulated by simulated microgravity. This was caused, at least partly, by decreased histone acetylation in macrophages under simulated microgravity [18].

The abundance of several metabolites in the cell culture supernatant of primary human macrophages is found to change under microgravity. Higher levels of 3-methyl-2-oxovaleric acid (3-methyl-2-pentanoic acid), benzoic acid, glycerol-3phosphate, ketoleucine (4-methyl-2-pentanoic acid) and fucose, and lower level of $\mathrm{N}$-acetyltryptophan are found in the microgravity condition, compared with the $1 * \mathrm{~g}$ control condition [19].

\section{Cytokine Secretion}

Cytokines are secretable proteins that function as the mediators and regulators of immune responses and the stimulators of hematopoiesis. Increased secretion of cytokines (e.g. IL-1 $\beta$, IL-2, IL-8, MCP-1, M-CSF, TGF- $\beta 1$, etc.) has been found in U937 cells cultured under simulated microgravity. The underlying mechanisms might involve an inhibition of intracellular cytokine degradation, as the proteasome activity in U937 cells under simulated microgravity has been shown to be reduced [8]. Increased NF-KappaB activity under microgravity may represent an additional mechanism. NF-KappaB (nuclear factor kappa-light-chain-enhancer of activated B cells) activates the transcription of cytokine genes [20]. When the murine macrophage cell line, RAW 264.7, was cultured under simulated microgravity, the concentration of nuclear NFKappaB has been reported to be increased [21].

Less IL-1 $\beta$ (Interleukin-1 $\beta$ ) is produced when the human monocytic cell lines, THP1 and U937, are stimulated with phorbol esters in space $[22,23]$. Protein kinase C (PKC) is known to be a cellular target of phorbol esters. When bound by Phorbol esters, PKC translocates from cytosol to cell membranes to acquire an active state and initiate downstream signaling [24], which may increase the expression of IL-1 $\beta$ [25]. PKC translocation from the cytosol fraction to the particulate fraction (which includes the nucleus, membrane, and insoluble cytoskeleton) in phorbol esters-stimulated U937 cells has been found to be inhibited in space [11,25]. This may at least partly account for the reduced phorbol esters-induced IL$1 \beta$ production in space.

When RAW 264.7 macrophage cells are treated with LPS (lipopolysaccharides; constituents of the outer membrane of Gram-negative bacteria) plus IFN- $\gamma$ (Interferon-gamma) under simulated microgravity, less NO (nitric oxide), TNF (Tumor Necrosis Factor)- $\alpha$, IL- 6 and IL-12 were shown to be secreted, compared with under $1 * \mathrm{~g}$ condition [26]. In our recent study, consistent results were found. The LPS-induced TNF- $\alpha$ 
expression in RAW 264.7 cells and primary mouse macrophages were decreased under simulated microgravity [27]. Molecular mechanism investigation revealed that neither TLR4 (Toll-like receptor 4, the receptor for LPS) expression nor the signal transduction downstream of TLR4 was inhibited under simulated microgravity. Furthermore, TNF- $\alpha$ mRNA stability did not decrease under simulated microgravity. Instead, we found that heat shock factor-1 (HSF1), a known repressor of TNF- $\alpha$ promoter, was markedly activated under simulated microgravity. Microgravity inhibition of LPS-induced TNF- $\alpha$ expression may be mediated by the microgravity-activated HSF1 [27]. In another study, we found that the LPS-induced IL-12B expression in primary mouse macrophages was decreased under simulated microgravity, in which activation of the $\mathrm{p} 38 \mathrm{MAPK}-\mathrm{C} / \mathrm{EBP} \beta$ pathway may play a role since $\mathrm{C} / \mathrm{EBP} \beta$ is a negative regulator of IL-12B transcription [28].

\section{Production of reactive oxygen species (ROS)}

Upon exposure to microbes, macrophages produce large amounts of ROS which have microbicidal effects [29]. When NR8383 macrophage cells were treated with zymosan (a component of fungal cell wall) under simulated microgravity, less ROS were reported to be produced, compared with under $1 * \mathrm{~g}$ condition [30]. This was suggested to be caused by diminished Syk phosphorylation under simulated microgravity [30], because Syk tyrosine kinase is a key signaling molecule for the zymosan-induced production of ROS by macrophages $[31,32]$. Reduced ROS production by macrophages under microgravity has also been observed when opsonized zymosan particles were used to stimulate the macrophage cells [33]. However, the inhibitory effect of microgravity on ROS production in macrophages is reported to only last for a short period (less than one minute) [34].

\section{Cell Migration}

Monocytes are derived from progenitors in the bone marrow and traffic via the blood stream to peripheral tissues, where they differentiate into macrophages and dendritic cells. In the condition of local inflammation, monocytes in the blood stream migrate to the site of inflammation and participate in local immune responses. Locomotion of $\mathrm{J} 111$ cells on gold particle-coated glass slides was shown to be reduced under no matter real or simulated microgravity $[6,7]$. In general, cell locomotion comprises three components, namely, protrusion of the leading edge, adhesion of the leading edge followed by deadhesion at the rear of the cell, and cytoskeletal contraction to pull the cell forward [35]. As the protrusion of the leading edge is driven by actin polymerization, the reduced $\beta$-actin expression under microgravity [8] might decelerate actin polymerization and partly account for the impaired cell locomotion in this environment. Secondly, the adhesion of the leading edge functions in preventing the retraction of the leading edge; the abnormality in the structure of focal adhesions $[6,7]$ suggests that the mechanic properties of focal adhesions might have defects, which could impair the firm adhesion of the leading edge and decrease cell motility. This inference is consistent with the observation that J111 cells under microgravity have shorter protrusions than under $1^{*} \mathrm{~g}[6,7]$. Lastly, PKC (protein kinase C) signaling plays a non-redundant role in cell locomotion [36]; the impaired PKC signaling under microgravity $[11,25]$ might also contribute to the decreased cell motility.

\section{Osteoclastogenesis}

More osteoclast-like plurinucleated cells are shown to be formed when the preosteoclastic FLG 29.1 cells are cultured with bone slices under simulated microgravity compared with under $1 * \mathrm{~g}$. The expression of TRAP (tartrate resistant acid phosphatase), an osteoclast-specific enzyme, is reported to be higher in the cells cultured under simulated microgravity [10]. Similarly, when bone marrow macrophages were cultured with bonelike scaffolds, M-CSF (Macrophage colony-stimulating factor) and RANKL (Receptor activator of nuclear factor kappa-B ligand) in space, more numerous and larger osteoclasts were formed compared with the ground control. The cells cultured in space expressed higher levels of TRAP and cathepsin K, which are osteoclast-specific enzymes [37]. These findings suggest that the microgravity environment could enhance osteoclastogenesis. Consistently, the number of osteoclasts per square millimeter of the trabecular surface is documented to be significantly increased in spaceflight rats [38].

Higher S100A8 expression is found in RAW 264.7 cells undergoing RANKL-stimulated osteoclastogenesis under simulated microgravity, compared with under $1 * \mathrm{~g}$ condition. siRNA knockdown of S100A8 expression in the RAW 264.7 cells under simulated microgravity is shown to inhibit RANKLstimulated osteoclastogenesis, indicating that microgravity can enhance osteoclastogenesis through S100A8 up-regulation [39]. It has been shown that murine S100A8 could be secreted by activated monocytes and macrophages and has potent chemotactic activity for monocytes [40]. Therefore, the microgravity-induced S100A8 overexpression might enhance osteoclastogenesis through promoting cell gathering and fusion. In addition, the fusion protein, Syncytin-1, is up-regulated by simulated microgravity. siRNA knockdown of Syncytin-1 expression in the RAW 264.7 cells under simulated microgravity inhibit RANKL-stimulated osteoclastogenesis, indicating that microgravity can enhance osteoclastogenesis through Syncytin-1 up-regulation as well [41].

Higher cytosolic calcium $\left(\mathrm{Ca}^{2+}\right)$ level and increased activation of the transcription factors, CREB (c-AMP responsive element-binding protein) and NFATc1 (Nuclear factor of activated T-cells, cytoplasmic 1), have been found in RAW 264.7 cells under simulated microgravity [39, 42]. These results indicate that the $\mathrm{Ca}^{2+-}$ Calmodulin kinases-CREB and $\mathrm{Ca}^{2+}$-Calcineurin-NFATc1 pathways are hyperactivated under simulated microgravity, which may increase the expression of osteoclast-specific genes and enhance osteoclastogenesis [43]. In addition, activation of MAP kinases (p38 and ERK) and NFKappaB has been found in RAW 264.7 cells under simulated microgravity [42, 21]. The activation of NF-KappaB and MAPK (Mitogen-activated protein kinases) signaling pathways is involved in the driving of osteoclastogenesis [44]. Therefore, microgravity might enhance osteoclastogenesis through increasing the activation of NF-KappaB and MAPK signaling pathways.

Tumor necrosis factor-related apoptosis inducing ligand (TRAIL) has been shown to increase osteoclastogenesis through a TRAF6 (TNF receptor associated factor 6) dependent signaling pathway. The expression of TRAIL in RAW 264.7 cells is reported to be up-regulated by simulated microgravity. A neutralizing antibody against TRAIL has been shown to reduce RANKL-stimulated osteoclastogenesis significantly under simulated microgravity, indicating that microgravity can enhance osteoclastogenesis through TRAIL up-regulation [45]. 


\section{DISCUSSION}

Previous studies concerning the microgravity effects on monocyte/macrophage physiology have revealed several dysfunctions of these cell types under microgravity (Table 1), which have implications for the human health in space. The inhibited development and proliferation under microgravity may reduce the normal reservoir of these cell types. The reduced production of ROS and proinflammatory cytokines by activated monocyte/macrophages under microgravity may limit the extent of both innate and adaptive immune responses in the context of bacteria or fungi infection, which could result in failure of pathogen control. The inhibited cell locomotion under microgravity may delay the recruitment of these cells to sites of local inflammation, which could result in delayed resolution of the inflammation. The enhanced osteoclastogenesis under microgravity, in combination with the enhanced bone resorption function of osteoclasts under microgravity [46, 37], may underlie the observed osteoporosis in space.
Moreover, considering that macrophages are professional antigen-presenting cells [47], it should be noted that microgravity might modulate the efficacy of antigen presentation by macrophages in the immune response to vaccines in several aspects, as follws. Firstly, microgravity may reduce the normal reservoir of macrophages. Secondly, microgravity may delay the recruitment of macrophages to the site of immune reaction to vaccines. Thirdly, microgravity may decrease the expression of MHC II molecules in macrophages, which may potentially decrease the efficacy of antigen presentation by macrophages.

More studies are required to fully elucidate the molecular mechanisms by which microgravity interferes with the monocyte/macrophage physiology as well as to what extent the immune responses to different vaccines would be affected in the microgravity environment, and to find measures that could counteract such adverse effects.

Table 1. Summary of the microgravity effects on monocyte/macrophage physiology

\begin{tabular}{ccc}
\hline Effects & Molecular mechanisms ${ }^{1}$ & References
\end{tabular}

1. Impaired development

2. Altered cytoskeleton structures

3. Inhibited growth

4. Decreased MHC II expression

5. Increased secretion of cytokines at the steady state

6. Decrease in phorbol estersinduced IL-1 production

7. Decrease in LPS-induced TNF- $\alpha$ expression

8. Decrease in LPS-induced

IL-12B expression

9. Decrease in LPS plus IFN$\gamma$-induced production of NO (nitric oxide) and IL-6

10. Decrease in zymosaninduced production of reactive oxygen species (ROS)

11. Inhibited cell locomotion

12. Enhanced

osteoclastogenesis
Reduced expression of genes in the ERK and NF-KappaB signaling pathways

Reduced cdc25B expression; Abnormalities in the expression and structure of microfilaments and microtubules

Decreased histone acetylation

$[6-8,10-12]$

Inhibition of intracellular cytokine degradation; Increased NF-KappaB activity

Impaired PKC signaling

$[11,22,23,25]$

Activation of heat shock factor-1 (a repressor of TNF- $\alpha$ promoter)

Activation of the $\mathrm{p} 38$ MAPK-C/EBP $\beta$ pathway

Diminished Syk phosphorylation

Reduced $\beta$-actin expression; The abnormality in the structure of focal adhesions; Impaired PKC signaling S100A8 overexpression; Syncytin-1 overexpression; Activation of $\mathrm{Ca}^{2+}-$

$[10,21,37,38$, Calmodulin kinases-CREB and $\mathrm{Ca}^{2+}{ }_{-}$Calcineurin-NFATc1 pathways; Activation of NF-KappaB and MAPK (Mitogen-activated protein kinases) signaling

$39,41,42,45]$ pathways; TRAIL overexpression 


\section{ACKNOWLEDGMENTS}

We thank Prof. Yong Zhao and Prof. Meifu Feng for their advice. This work was supported by grants from Guangxi Provincial Department of Education (KY2015LX281) and Guangxi Provincial Department of Science and Technology (2015GXNSFBA139122).

\section{CONFLICT OF INTEREST}

The authors declare that they have no conflict of interest.

\section{REFERENCES}

1. Hughes-Fulford M. To infinity ... and beyond! Human spaceflight and life science. Faseb J. 2011;25(9):2858-64. doi:10.1096/fj.11-0902ufm.

2. Blaber E, Marcal H, Burns BP. Bioastronautics: the influence of microgravity on astronaut health. Astrobiology. 2010;10(5):463-73. doi:10.1089/ast.2009.0415

3. Armstrong JW, Gerren RA, Chapes SK. The effect of space and parabolic flight on macrophage hematopoiesis and function. Experimental cell research. 1995;216(1):160-8. doi:10.1006/excr.1995.1020.

4. Racine RN, Cormier SM. Effect of spaceflight on rat hepatocytes: a morphometric study. J Appl Physiol. 1992;73(2 Suppl):136S-41S.

5. Shi L, Tian H, Wang P, Li L, Zhang Z, Zhang J et al. Spaceflight and simulated microgravity suppresses macrophage development via altered RAS/ERK/NFkB and metabolic pathways. Cell Mol Immunol. 2020. doi:10.1038/s41423-019-0346-6.

6. Meloni MA, Galleri G, Pani G, Saba A, Pippia P, Cogoli-Greuter M. Space flight affects motility and cytoskeletal structures in human monocyte cell line J-111. Cytoskeleton (Hoboken). 2011;68(2):125-37.

7. Meloni MA, Galleri G, Pippia P, Cogoli-Greuter M. Cytoskeleton changes and impaired motility of monocytes at modelled low gravity. Protoplasma. 2006;229(2-4):243-9. doi:10.1007/s00709-006-0210-2.

8. Maier JA. Impact of simulated microgravity on cell cycle control and cytokine release by U937 cells. International journal of immunopathology and pharmacology. 2006;19(2):279-86.

9. Drexler HG. Monocytic cell lines. In: Masters J, Palsson B, editors. Human Cell Culture Volume III. New York: Kluwer Academic; 2002. p. 237-57.

10. Monici M, Fusi F, Paglierani M, Marziliano N, Cogoli A, Pratesi R et al. Modeled gravitational unloading triggers differentiation and apoptosis in preosteoclastic cells. Journal of cellular biochemistry. 2006;98(1):65-80. doi:10.1002/jcb.20747.

11. Hatton JP, Gaubert F, Lewis ML, Darsel Y, Ohlmann P, Cazenave JP et al. The kinetics of translocation and cellular quantity of protein kinase $\mathrm{C}$ in human leukocytes are modified during spaceflight. Faseb J. 1999;13 Suppl:S23-33

12. Villa A, Versari S, Maier JA, Bradamante S. Cell behavior in simulated microgravity: a comparison of results obtained with RWV and RPM. Gravitational and space biology bulletin : publication of the American Society for Gravitational and Space Biology. 2005;18(2):89-90.

13. Lammer C, Wagerer S, Saffrich R, Mertens D, Ansorge W, Hoffmann I. The cdc25B phosphatase is essential for the G2/M phase transition in human cells. Journal of cell science. 1998;111 ( Pt 16):2445-53.

14. Civelekoglu-Scholey G, Scholey JM. Mitotic force generators and chromosome segregation. Cellular and molecular life sciences : CMLS. 2010;67(13):2231-50. doi:10.1007/s00018-010-0326-6.

15. Fededa JP, Gerlich DW. Molecular control of animal cell cytokinesis. Nature cell biology. 2012;14(5):440-7. doi:10.1038/ncb2482.

16. Maccarrone M, Battista N, Meloni M, Bari M, Galleri G, Pippia P et al. Creating conditions similar to those that occur during exposure of cells to microgravity induces apoptosis in human lymphocytes by 5 -lipoxygenasemediated mitochondrial uncoupling and cytochrome c release. Journal of leukocyte biology. 2003;73(4):472-81

17. Paulsen K, Tauber S, Dumrese C, Bradacs G, Simmet DM, Golz N et al. Regulation of ICAM-1 in cells of the monocyte/macrophage system in microgravity. BioMed research international. 2015;2015:538786. doi:10.1155/2015/538786.

18. Wang C, editor. An epigenetic mechanism for decreased MHC II expression in macrophages under simulated microgravity. 69th
International Astronautical Congress: \#InvolvingEveryone, IAC 2018; 2018: International Astronautical Federation, IAF.

19. Tauber S, Lauber BA, Paulsen K, Layer LE, Lehmann M, Hauschild S et al. Cytoskeletal stability and metabolic alterations in primary human macrophages in long-term microgravity. PLoS ONE. 2017;12(4). doi:10.1371/journal.pone.0175599.

20. Krakauer T. Nuclear factor-kappaB: fine-tuning a central integrator of diverse biologic stimuli. International reviews of immunology. 2008;27(5):286-92. doi:10.1080/08830180802317957

21. Zwart SR, Pierson D, Mehta S, Gonda S, Smith SM. Capacity of omega-3 fatty acids or eicosapentaenoic acid to counteract weightlessnessinduced bone loss by inhibiting NF-kappaB activation: from cells to bed rest to astronauts. Journal of bone and mineral research : the official journal of the American Society for Bone and Mineral Research. 2010;25(5):104957. doi:10.1359/jbmr.091041.

22. Limouse M, Manie S, Konstantinova I, Ferrua B, Schaffar L. Inhibition of phorbol ester-induced cell activation in microgravity. Experimental cell research. 1991;197(1):82-6.

23. Schmitt DA, Hatton JP, Emond C, Chaput D, Paris H, Levade T et al. The distribution of protein kinase $\mathrm{C}$ in human leukocytes is altered in microgravity. FASEB J. 1996;10(14):1627-34.

24. Newton AC. Protein kinase C: poised to signal. American journal of physiology Endocrinology and metabolism. 2010;298(3):E395-402. doi:10.1152/ajpendo.00477.2009.

25. Hatton JP, Gaubert F, Cazenave JP, Schmitt D. Microgravity modifies protein kinase $\mathrm{C}$ isoform translocation in the human monocytic cell line U937 and human peripheral blood T-cells. Journal of cellular biochemistry. 2002;87(1):39-50. doi:10.1002/jcb.10273.

26. Hsieh CL, Chao PD, Fang SH. Morin sulphates/glucuronides enhance macrophage function in microgravity culture system. European journal of clinical investigation. 2005;35(9):591-6. doi:10.1111/j.13652362.2005.01551.x.

27. Wang C, Luo H, Zhu L, Yang F, Chu Z, Tian H et al. Microgravity inhibition of lipopolysaccharide-induced tumor necrosis factor-alpha expression in macrophage cells. INFLAMM RES. 2014;63(1):91-8. doi:10.1007/s00011-013-0676-2.

28. Wang C, Chen H, Luo H, Zhu L, Zhao Y, Tian H et al. Microgravity activates p38 MAPK-C/EBPbeta pathway to regulate the expression of arginase and inflammatory cytokines in macrophages. INFLAMM RES. 2015;64(5):303-11. doi:10.1007/s00011-015-0811-3.

29. Lambeth JD, Neish AS. Nox enzymes and new thinking on reactive oxygen: a double-edged sword revisited. Annual review of pathology. 2014;9:119-45. doi:10.1146/annurev-pathol-012513-104651.

30. Brungs S, Kolanus W, Hemmersbach R. Syk phosphorylation - a gravisensitive step in macrophage signalling. Cell communication and signaling : CCS. 2015;13:9. doi:10.1186/s12964-015-0088-8.

31. Underhill DM, Rossnagle E, Lowell CA, Simmons RM. Dectin-1 activates Syk tyrosine kinase in a dynamic subset of macrophages for reactive oxygen production. Blood. 2005;106(7):2543-50. doi:10.1182/blood-2005-03-1239.

32. Mocsai A, Ruland J, Tybulewicz VL. The SYK tyrosine kinase: a crucial player in diverse biological functions. Nature reviews Immunology. 2010;10(6):387-402. doi:10.1038/nri2765.

33. Adrian A, Schoppmann K, Sromicki J, Brungs S, von der Wiesche M, Hock B et al. The oxidative burst reaction in mammalian cells depends on gravity. Cell communication and signaling : CCS. 2013;11:98. doi:10.1186/1478-811X-11-98.

34. Thiel CS, De Zélicourt D, Tauber S, Adrian A, Franz M, Simmet DM et al. Rapid adaptation to microgravity in mammalian macrophage cells. Sci Rep. 2017;7(1). doi:10.1038/s41598-017-00119-6.

35. Ananthakrishnan R, Ehrlicher A. The forces behind cell movement. International journal of biological sciences. 2007;3(5):303-17.

36. Larsson $\mathrm{C}$. Protein kinase $\mathrm{C}$ and the regulation of the actin cytoskeleton. Cellular signalling. 2006;18(3):276-84. doi:10.1016/j.cellsig.2005.07.010. 37. Tamma R, Colaianni G, Camerino C, Di Benedetto A, Greco G, Strippoli $\mathrm{M}$ et al. Microgravity during spaceflight directly affects in vitro osteoclastogenesis and bone resorption. Faseb J. 2009;23(8):2549-54. doi:10.1096/fj.08-127951.

38. Vico L, Chappard D, Alexandre C, Palle S, Minaire P, Riffat G et al. Effects of weightlessness on bone mass and osteoclast number in pregnant rats after a five-day spaceflight (COSMOS 1514). Bone. 1987;8(2):95-103. 39. Sambandam Y, Blanchard JJ, Daughtridge G, Kolb RJ, Shanmugarajan $\mathrm{S}$, Pandruvada $\mathrm{SN}$ et al. Microarray profile of gene expression during osteoclast differentiation in modelled microgravity. Journal of cellular biochemistry. 2010;111(5):1179-87. doi:10.1002/jcb.22840. 
40. Goyette J, Geczy CL. Inflammation-associated S100 proteins: new mechanisms that regulate function. Amino acids. 2011;41(4):821-42. doi:10.1007/s00726-010-0528-0.

41. Ethiraj P, Link JR, Sinkway JM, Brown GD, Parler WA, Reddy SV. Microgravity modulation of syncytin-A expression enhance osteoclast formation. Journal of cellular biochemistry. 2018;119(7):5696-703. doi:10.1002/jcb.26750.

42. Saxena R, Pan G, Dohm ED, McDonald JM. Modeled microgravity and hindlimb unloading sensitize osteoclast precursors to RANKL-mediated osteoclastogenesis. Journal of bone and mineral metabolism. 2011;29(1):111-22. doi:10.1007/s00774-010-0201-4.

43. Shinohara M, Takayanagi $\mathrm{H}$. Novel osteoclast signaling mechanisms. Current osteoporosis reports. 2007;5(2):67-72.
44. Edwards JR, Mundy GR. Advances in osteoclast biology: old findings and new insights from mouse models. Nature reviews Rheumatology. 2011;7(4):235-43. doi:10.1038/nrrheum.2011.23.

45. Sambandam Y, Baird KL, Stroebel M, Kowal E, Balasubramanian S, Reddy SV. Microgravity Induction of TRAIL Expression in Preosteoclast Cells Enhances Osteoclast Differentiation. Scientific reports. 2016;6:25143. doi:10.1038/srep25143.

46. Nabavi N, Khandani A, Camirand A, Harrison RE. Effects of microgravity on osteoclast bone resorption and osteoblast cytoskeletal organization and adhesion. Bone. 2011;49(5):965-74 doi:10.1016/j.bone.2011.07.036.

47. Neefjes J, Jongsma ML, Paul P, Bakke O. Towards a systems understanding of MHC class I and MHC class II antigen presentation. Nature reviews Immunology. 2011;11(12):823-36. doi:10.1038/nri3084. 